\title{
Bur open A systematic review of evidence on the links between patient experience and clinical safety and effectiveness
}

\author{
Cathal Doyle, ${ }^{1}$ Laura Lennox, ${ }^{1,2}$ Derek Bell ${ }^{1,2}$
}

To cite: Doyle C, Lennox L, Bell D. A systematic review of evidence on the links between patient experience and clinical safety and effectiveness. BMJ Open 2013:3:e001570.

doi:10.1136/bmjopen-2012001570

- Prepublication history and additional material for this paper are available online. To view these files please visit the journal online (http://dx.doi.org/10.1136/ bmjopen-2012-001570).

Received 18 June 2012 Revised 2 November 2012 Accepted 12 November 2012

This final article is available for use under the terms of the Creative Commons Attribution Non-Commercial 2.0 Licence; see http://bmjopen.bmj.com

${ }^{1}$ NIHR CLAHRC for North West London, Chelsea and Westminster Hospital, London, UK

${ }^{2}$ Department of Medicine, Imperial College London, Chelsea and Westminster Hospital, London, UK

Correspondence to Dr Cathal Doyle; c.doyle@imperial.ac.uk

\section{ABSTRACT}

Objective: To explore evidence on the links between patient experience and clinical safety and effectiveness outcomes.

Design: Systematic review.

Setting: A wide range of settings within primary and secondary care including hospitals and primary care centres.

Participants: A wide range of demographic groups and age groups.

Primary and secondary outcome measures: A broad range of patient safety and clinical effectiveness outcomes including mortality, physical symptoms, length of stay and adherence to treatment.

Results: This study, summarising evidence from 55 studies, indicates consistent positive associations between patient experience, patient safety and clinical effectiveness for a wide range of disease areas, settings, outcome measures and study designs. It demonstrates positive associations between patient experience and self-rated and objectively measured health outcomes; adherence to recommended clinical practice and medication; preventive care (such as health-promoting behaviour, use of screening services and immunisation); and resource use (such as hospitalisation, length of stay and primary-care visits). There is some evidence of positive associations between patient experience and measures of the technical quality of care and adverse events. Overall, it was more common to find positive associations between patient experience and patient safety and clinical effectiveness than no associations.

Conclusions: The data presented display that patient experience is positively associated with clinical effectiveness and patient safety, and support the case for the inclusion of patient experience as one of the central pillars of quality in healthcare. It supports the argument that the three dimensions of quality should be looked at as a group and not in isolation. Clinicians should resist sidelining patient experience as too subjective or mood-oriented, divorced from the 'real' clinical work of measuring safety and effectiveness.

\section{INTRODUCTION}

Patient experience is increasingly recognised as one of the three pillars of quality in healthcare alongside clinical effectiveness and

\section{ARTICLE SUMMARY}

Article focus

- Should patient experience, as advocated by the Institute of Medicine and the NHS Outcomes Framework, be seen as one of the pillars of quality in healthcare alongside patient safety and clinical effectiveness?

- What aspects of patient experience can be linked to clinical effectiveness and patient safety outcomes?

- What evidence is available on the links between patient experience and clinical effectiveness and patient safety outcomes?

Key messages

- The results show that patient experience is consistently positively associated with patient safety and clinical effectiveness across a wide range of disease areas, study designs, settings, population groups and outcome measures.

- Patient experience is positively associated with self-rated and objectively measured health outcomes; adherence to recommended medication and treatments; preventative care such as use of screening services and immunisations; healthcare resource use such as hospitalisation and primary-care visits; technical quality-of-care delivery and adverse events.

- This study supports the argument that patient experience, clinical effectiveness and patient safety are linked and should be looked at as a group.

Strengths and limitations of this study

- This study demonstrates an approach to designing a systematic review for the 'catch-all' term patient experience, and brings together evidence from a variety of sources that may otherwise remain dispersed.

- This was a time-limited review and there is scope to expand this search based on the results and broaden the search terms to uncover further evidence.

patient safety. ${ }^{1}$ In the NHS, the measurement of patient experience data to identify strengths and weaknesses of healthcare delivery, drive-quality improvement, inform 
commissioning and promote patient choice is now mandatory. ${ }^{2-4}$ In addition to data on harm avoidance or success rates for treatments, providers are now assessed on aspects of care such as dignity and respect, compassion and involvement in care decisions. ${ }^{4}$ In England, these data are published in Quality Accounts and the Commissioning for Quality and Innovation payment framework which makes a proportion of care providers' income conditional on the improvement in this domain. ${ }^{5}$

The inclusion of patient experience as a pillar of quality is often justified on grounds of its intrinsic value-that the expectation of humane, empathic care is requires no further justification. It is also justified on more utilitarian grounds as a means of improving patient safety and clinical effectiveness. ${ }^{6} 7$ For example, clear information, empathic, two-way communication and respect for patients' beliefs and concerns could lead to patients being more informed and involved in decision-making and create an environment where patients are more willing to disclose information. Patients could have more 'ownership' of clinical decisions, entering a 'therapeutic alliance' with clinicians. This could support improved and more timely diagnosis, clinical decisions and advice and lead to fewer unnecessary referrals or diagnostic tests. ${ }^{8}{ }^{9}$ Increased patient agency can encourage greater participation in personal care, compliance with medication, adherence to recommended treatment and monitoring of prescriptions and dose. $^{9}{ }^{10}$ Patients can be informed about what to expect from treatment and be motivated to report adverse events or complications and keep a list of their medical histories, allergies and current medications. ${ }^{11}$

Patients' direct experience of care process through clinical encounters or as an observer (eg, as a patient on a hospital ward) can provide valuable insights into everyday care. Examples include attention to pain control, assistance with bathing or help with feeding, the environment (cleanliness, noise and physical safety) and coordination of care between professions or organisations. Given the organisational fragmentation of much of healthcare and the numerous services with which many patients interact, the measurement of patient experience may help provide a 'whole-system' perspective not readily available from more discrete patient safety and clinical effectiveness measures. ${ }^{11}$

Focusing on such utilitarian arguments, this study reviews evidence on links that have been demonstrated between patient experience and clinical effectiveness and patient safety.

\section{METHODS}

Identifying variables relevant to patient experience

Patient experience is a term that encapsulates a number of dimensions, and in preliminary database searches, this phrase, on its own, uncovered a limited number of useful studies. To broaden and structure the search for evidence, identify search terms and provide a framework for analysis, it was necessary to identify what patient experience entails and outline potential mechanisms through which it is proposed to impact on safety and effectiveness. As such, we combined common elements from patient experience frameworks produced by The Institute of Medicine, ${ }^{1}$ Picker Institute ${ }^{12}$ and NICE. ${ }^{13}$

Table 1 delineates different dimensions of patient experience and distinguishes between 'relational' and 'functional' aspects. ${ }^{10}{ }^{14}$ Relational aspects refer to interpersonal aspects of care-the ability of clinicians to empathise, respect the preferences of patients, include them in decision-making and provide information to enable self-care. ${ }^{10}$ It also refers to patients' expectations that professionals will put their interest above other considerations and be honest and transparent when something goes wrong. ${ }^{8}{ }^{15}$ Functional aspects relate to basic expectations about how care is delivered, such as attention to physical needs, timeliness of care, clean and safe environments, effective coordination between professionals, and continuity.

Using these frameworks and discursive documents in this area of research ${ }^{9} 101617$ as a guide, we identified

Table 1 Identifying aspects of patient experience and search terms

\section{Relational aspects}

Emotional and psychological support, relieving fear and anxiety, treated with respect, kindness, dignity, compassion, understanding

Participation of patient in decisions and respect and understanding for beliefs, values, concerns, preferences and their understanding of their condition

Involvement of, and support for family and carers in decisions

Clear, comprehensible information and communication tailored to patient needs to support informed decisions (awareness of available options, risks and benefits of treatments) and enable self-care

Transparency, honesty, disclosure when something goes wrong

\section{Functional aspects}

Effective treatment delivered by trusted professionals

Timely, tailored and expert management of physical symptoms

Attention to physical support needs and environmental needs (eg, clean, safe, comfortable environment)

Coordination and continuity of care; smooth transitions from one setting to another 


\section{Box 1 Search terms denoting patient experience}

Patient-centred care; patient engagement; clinical interaction; patientclinician; clinician-patient; patient-doctor; doctor-patient; physician-patient; patient-physician; patient-provider; interpersonal treatment; physician discussion; trust in physician; empathy; compassion; respect; responsiveness; patient preferences; shared decision-making; therapeutic alliance; participation in decisions; decision-making; autonomy; caring; kindness; dignity; honesty; participation; right to decide; physical comfort; involvement (of family, carers, friends); emotional support; continuity (of care); smooth transition; emotional support.

words and phrases commonly used to denote aspects of patient experience, examples of which are listed in box 1 .

These were combined with search terms representing patient safety and clinical effectiveness outcomes, hypothesised to be associated with patient experience in discursive literature. We searched for a broad range of outcome measures, including both self-rated and 'objective' measurements of health status, physical health and mental health and well-being, the use of preventive health services, compliance or adherence to healthpromoting behaviour and resource use.

Combining these two sets of search terms in the EMBASE database, we identified 5323 papers whose abstracts were then reviewed. If deemed relevant, the full article was retrieved to assess whether it met the inclusion criteria.

Given concerns about the sole use of protocol-driven search strategies for complex evidence ${ }^{18}$ for the full-text articles retrieved for review, we used a 'snowballing' approach to identify further studies. This involved sourcing further articles in these studies for assessment and using the 'related articles' function in the Pubmed database. We repeated this for new articles identified until the approach ceased to identify new studies.

\section{Inclusion criteria, assessment of quality} and categorisation of evidence

We included studies that measured associations between patients' reporting of their experience and patient safety and clinical effectiveness outcomes. These included studies measuring associations between patient experience and safety or effectiveness outcomes either at a patient level (ie, data on both types of variables for the same patients) or at an organisational level (ie, associations between aggregated measures of patient experience and safety and effectiveness outcomes for the same type of organisation such as a hospital or primary-care practice).

We included studies where the variables denoting patient experience and patient safety and clinical effectiveness were measured in a credible way, through the use of validated tools. For patient experience variables, these include surveys covering several aspects of experience (such as Picker surveys and the Hospital Consumer Assessment of Healthcare Providers and Systems survey) and specific aspects (such as a 'Working Alliance Scale', ${ }^{19}$ Multidimensional Health Locus of Control Scale scale ${ }^{20}$ or Usual Provider Continuity index ${ }^{21}$ ). For patient safety and clinical effectiveness, these include, for example, generic health and quality of life surveys (such as Short-Form 36), disease-specific surveys (such as the Seattle Angina Questionnaire $^{22}$ ), measures of the technical quality of care (such as the Hospital Quality Alliance (HQA) score), reviews of medical records and care provider data. ${ }^{23}$ Details of the methods used to measure variables in each study are included in tables 5 and 6 .

We included studies where the sample size of patients or organisations appeared sufficiently large to conduct a meaningful statistical analysis (excluding studies with fewer than 50 subjects). When extracting data relevant to our study from systematic reviews, we selected only those studies that met these criteria.

We then searched the studies' results for positive associations (where a better patient experience is associated with safer or more effective care), negative associations (where a better patient experience is associated with less safe or less effective care) and no associations. Associations refer to cases where one measure of patient experience (typically an overall rating of patient experience for a care provider) has a statistically significant association with one or more clinical effectiveness or patient safety variable. If a study showed associations between several aspects of patient experience that appeared to be closely related (eg, 'listening', 'empathy', or 'respect') and an aspect of effectiveness or safety, this was counted as one association found. This was to avoid exaggerating the weight of the evidence by 'over counting' associations.

Two main types of studies emerged in the searchthose focusing on interventions to improve aspects of patient experience and those exploring associations between patient experience variables and patient safety and clinical effectiveness variables. To manage the scope of this time-limited review, we decided to restrict analysis of the large number of interventions to the evidence contained within systematic reviews.

\section{RESULTS}

Overall, the evidence indicates positive associations between patient experience and patient safety and clinical effectiveness that appear consistent across a range of disease areas, study designs, settings, population groups and outcome measures. Positive associations found outweigh 'no associations' by 429-127. Of the four studies where 'no associations' outweigh positive associations, there is no suggestion that these are methodologically superior. Negative associations were rare. Of the 40 individual studies assessed in table 5 negative associations (between patient experience of clinical team interactions and continuity of care and separate assessment of the quality of clinical care) were found in only one study. ${ }^{24}$ 


\begin{tabular}{lc} 
Table 2 Methods used to measure variables \\
\hline & $\begin{array}{l}\text { Number } \\
\text { of studies }\end{array}$ \\
\hline Patient experience variables & 31 \\
Survey & 2 \\
Interviews & 1 \\
Medical records & \\
Effectiveness and safety variables & 12 \\
Survey for self-rated healthcare & 14 \\
Other survey & 3 \\
Medical records & 3 \\
Data-monitoring quality of care & \\
delivery (eg, audit, HQA, HEDIS) & 3 \\
Care provider outcome data & 1 \\
Physical examination & 2 \\
Patient interviews & \\
\hline HQA, Hospital Quality Alliance; HEDIS, Healthcare Effectiveness \\
Data and Information Set.
\end{tabular}

Table 2 shows surveys to be the predominant method used to measure variables for individual studies (figure 1).

Table 3 presents the frequency of positive associations and 'no associations' categorised by type of outcomes (for 378 of the 556 cases where sufficient information was available to categorise). These include objectively measured health outcomes (eg, 'mortality', 'blood glucose levels', 'infections', 'medical errors'); selfreported health and well-being outcomes (eg, 'health status', 'functional ability' 'quality of life', 'anxiety'); adherence to recommended treatment and use of preventive care services likely to improve health outcomes (eg, 'medication compliance', 'adherence to treatment' and screening for a variety of conditions); outcomes related to healthcare resource use (eg, 'hospitalisations', 'hospital readmission', 'emergency department use', 'primary care visits'); errors or adverse events and measures of the technical quality of care.

Table 4 shows associations categorised by type of care provider (for the subset of studies focusing on one setting) and for studies focused on chronic conditions.

\section{Disease Areas}

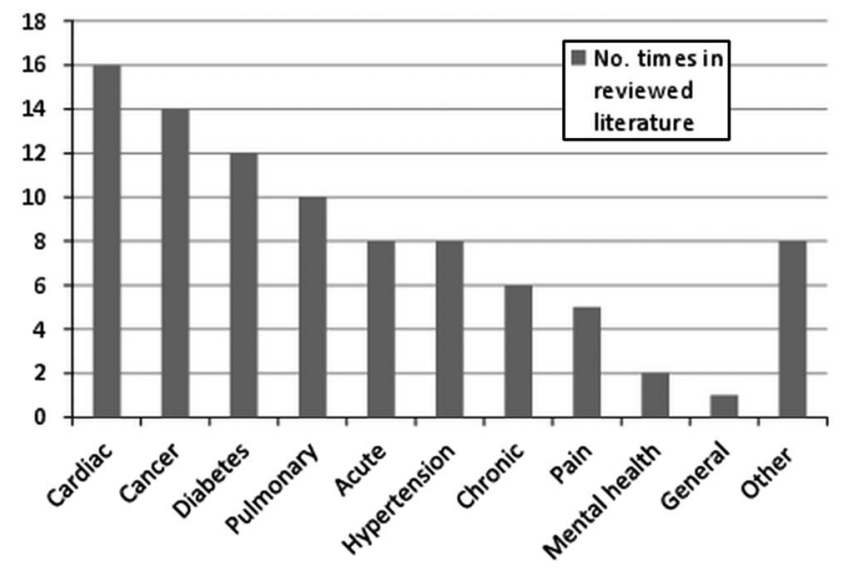

Figure 1 Outlines the disease areas covered.
Tables 5 and 6 present details of all studies identified, specifying the analytical focus of each study, methods to measure variables and positive associations and 'no asscoiations' found.

\section{DISCUSSION}

Overall, the evidence indicates associations between patient experience, clinical effectiveness and patient safety that appear consistent across a range of disease areas, study designs and settings.

As table 3 indicates, the evidence shows positive associations found outweigh those not found for both selfassessment of physical health and mental health (61 vs 36) and 'objective' measures of health outcomes (eg, where measures are taken by a clinician or by reviewing medical records) (29 vs 11). For objective measures, one study ${ }^{25}$ shows positive associations for ulcer disease, hypertension and breast cancer. Two studies on myocardial infarction show positive associations with survival 1 year after discharge ${ }^{26}$ and inpatient mortality. ${ }^{27}$ Objective measurement is less frequently explored than self-rated health and is an area that could benefit from further research.

Evidence is strong in the case of adherence to recommended medical treatment. A meta-analysis included in this study showed positive associations between the quality of clinician-patient communications and adherence to medical treatment in 125 of 127 studies analysed and showed the odds of patient adherence was 1.62 times higher where physicians had communication training. ${ }^{28}$ Regarding compliance with medication, positive associations found to outweigh those not found. ${ }^{20} 29-35$ A review of interventions to increase adherence to medication (not included in this study) showed communication of information, good provider-patient relationships and patients' agreement with the need for treatment as common determinants of effectiveness. ${ }^{36}$ There is evidence of better use of preventive services, such as screening services in diabetes, colorectal, breast and cervical cancer; cholesterol testing and immunisation. ${ }^{24} 25$ 37-39 There is also evidence of impacts on resource use of primary and secondary care (such as hospitalisations, readmissions and primary care visits) ${ }^{21} 2940-45$

For studies exploring associations between patient experience and technical quality of care measured by other means, the evidence is mixed. Two studies in acute care showed positive associations between overall ratings of patient experience and ratings of the technical quality of care (using HQA measures) for myocardial infarction, congestive heart failure, pneumonia and complications from surgery. ${ }^{23} 46$ Another found an association with adherence to clinical guidelines for acute myocardial infarction. ${ }^{27} \mathrm{~A}$ similar study in primary care found positive associations between patient experience of processes and measurement of care quality (from the Healthcare Effectiveness Data and Information Set (HEDIS) system measuring care quality for disease prevention and management in chronic conditions). ${ }^{24}$ 
Table 3 Associations categorised by type of outcome

\begin{tabular}{|c|c|c|c|c|c|c|c|c|}
\hline & $\begin{array}{l}\text { Objective' } \\
\text { health } \\
\text { outcomes }\end{array}$ & $\begin{array}{l}\text { Self-reported } \\
\text { health and } \\
\text { wellbeing }\end{array}$ & $\begin{array}{l}\text { Adherence } \\
\text { to } \\
\text { treatment } \\
\text { (including } \\
\text { medication) }\end{array}$ & $\begin{array}{l}\text { Preventive } \\
\text { care }\end{array}$ & $\begin{array}{l}\text { Healthcare } \\
\text { resource } \\
\text { use }\end{array}$ & $\begin{array}{l}\text { Adverse } \\
\text { events }\end{array}$ & $\begin{array}{l}\text { Technical } \\
\text { quality of } \\
\text { care }\end{array}$ & $\begin{array}{l}\text { All } \\
\text { categories }\end{array}$ \\
\hline $\begin{array}{l}\text { No of positive } \\
\text { associations found }\end{array}$ & 29 & 61 & 152 & 24 & 31 & 7 & 8 & 312 \\
\hline 'No associations' & 11 & 36 & 7 & 2 & 6 & 0 & 4 & 66 \\
\hline
\end{tabular}

However, two other studies found no associations between patients' ratings and ratings based on an assessment of medical records. ${ }^{4} 48$

Some studies show positive associations between patients' perspective or observations of processes of care and the safety of care recorded through other means. Isaac $^{46}$ found positive associations between ratings of patient experience and six patient-safety indicators (decubitus ulcer; failure to rescue; infections due to medical care; postoperative haemorrhage, respiratory failure, pulmonary embolism and sepsis). Two studies examining evidence for patients' ability to identify medical errors or adverse events in hospital showed positive associations between patients' accounts of their experience of adverse events and the documentation of events in medical records. ${ }^{49}{ }^{50}$ But another study shows only $2 \%$ of patient-reported errors were classified by medical reviewers as 'real clinical medical errors' with most 'reclassified' by clinicians as 'misunderstandings' or 'behaviour or communication problems'. 51 Overall, there is less evidence available on safety compared to effectiveness and this should be a priority for future research in this area.

Research from other studies not included in this review support these findings. For example, research on 'decision aids' to ensure that patients are well informed about their treatments, and that decisions reflect the preferences of patients indicates that patient engagement has a beneficial impact on outcomes. For example, awareness of the risks of surgical procedures resulted in a $23 \%$ reduction in surgical interventions and better functional status. ${ }^{52}$ Another review showed that provision of good information and emotional support are associated with better recovery from surgery and heart attacks. ${ }^{53}$

\begin{tabular}{|c|c|c|}
\hline $\begin{array}{l}\text { Weight of evidence } \\
\text { by provider and for } \\
\text { chronic conditions }\end{array}$ & $\begin{array}{l}\text { Associations } \\
\text { found }\end{array}$ & $\begin{array}{l}\text { No of } \\
\text { associations }\end{array}$ \\
\hline Primary care & 110 & 48 \\
\hline Hospital & 43 & 17 \\
\hline Chronic conditions & 53 & 9 \\
\hline
\end{tabular}

\section{STUDY STRENGTHS AND LIMITATIONS}

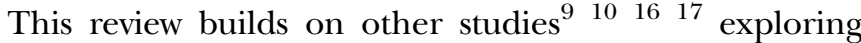
links between these three domains. This study also demonstrates an approach to designing a systematic search for evidence for the 'catch-all' term patient experience, bringing together evidence from a variety of sources that may otherwise remain dispersed. This approach can be used or adapted for further research in this area.

This was a time-limited review and there is scope to expand this search, based on our results. There may be scope to broaden the search terms and this may uncover further evidence. The first search was confined to one database and the review focused primarily on peerreviewed literature excluding grey literature. To manage the scope of this review, we restricted the analysis of interventions to improve patient experience to evidence within systematic reviews. While we used some quality criteria to filter studies (including the use of validated tools to measure experience, safety and effectiveness outcomes and sample size), with more time a more detailed formal quality assessment may have added value to the study. Although all positive associations included in the study are statistically significant, the strength of associations vary. Because of time constraints and the heterogeneity of measures used, we did not systematically compare the strengths of positive associations in different studies, but this may be an area for future work. There may also be scope to explore whether future research in this area could go beyond the counting of associations in this study through, for example, meta-analysis. As always, there may be a publication bias in favour of studies showing positive associations between patient experience variables and safety and effectiveness outcomes. ${ }^{54}$ In addition, 28 of the 40 individual studies assessed were conducted in the USA and caution is needed about their applicability to other healthcare systems.

\section{CONCLUSION}

The inclusion of patient experience as one of the pillars of quality is partly justified on the grounds that patient experience data, robustly collected and analysed, may help highlight strengths and weaknesses in effectiveness 


\begin{tabular}{|c|c|c|c|c|c|c|c|c|c|}
\hline Author & $\begin{array}{l}\text { Type of study, } \\
\text { sample size, } \\
\text { country }\end{array}$ & Setting & Disease focus & $\begin{array}{l}\text { Unit of } \\
\text { analysis } \\
\text { (patient } \\
\text { (P) or } \\
\text { org (O) }\end{array}$ & $\begin{array}{l}\text { Patient experience } \\
\text { focus and method } \\
\text { used }\end{array}$ & $\begin{array}{l}\text { Safety and } \\
\text { effectiveness } \\
\text { measure }\end{array}$ & $\begin{array}{l}\text { Association } \\
\text { demonstrated }\end{array}$ & $\begin{array}{l}\text { Association not } \\
\text { demonstrated }\end{array}$ & $\begin{array}{l}\text { Assoc. } \\
\text { Found } \\
\text { vs NOT } \\
\text { found }\end{array}$ \\
\hline Chang et $a f^{48}$ & $\begin{array}{l}\text { Cohort study, } \\
236 \text { patients, } \\
\text { USA }\end{array}$ & $\begin{array}{l}\text { Managed } \\
\text { care } \\
\text { organisation }\end{array}$ & $\begin{array}{l}22 \text { clinical } \\
\text { conditions }\end{array}$ & $P$ & $\begin{array}{l}\text { Providers } \\
\text { communication (The } \\
\text { Consumer Assessment } \\
\text { of Healthcare Providers } \\
\text { and Systems survey } \\
\text { and 'Quality of care') }\end{array}$ & $\begin{array}{l}\text { Technical quality and } \\
\text { patient global ratings } \\
\text { (medical records and } \\
\text { patient interviews) }\end{array}$ & None & $\begin{array}{l}\text { Technical quality } \\
\text { of care }\end{array}$ & $0 / 1$ \\
\hline $\begin{array}{l}\text { Sequist } \\
\text { et } a^{R^{4}}\end{array}$ & $\begin{array}{l}\text { Cross-sectional } \\
\text { study, } 492 \\
\text { settings, USA }\end{array}$ & Primary care & $\begin{array}{l}\text { Cervical, breast } \\
\text { and colorectal } \\
\text { cancer, } \\
\text { chlamydia, } \\
\text { cardiovascular } \\
\text { conditions, } \\
\text { asthma, } \\
\text { diabetes }\end{array}$ & $\mathrm{P}$ & $\begin{array}{l}\text { Doctor-patient } \\
\text { communication, clinical } \\
\text { team interactions, } \\
\text { organisational features } \\
\text { of care (The } \\
\text { Ambulatory Care } \\
\text { Experiences survey) }\end{array}$ & $\begin{array}{l}\text { Clinical quality focusing } \\
\text { on disease prevention, } \\
\text { disease management } \\
\text { and outcomes of care } \\
\text { (Healthcare } \\
\text { Effectiveness Data and } \\
\text { Information Set } \\
\text { (HEDIS)) }\end{array}$ & $\begin{array}{l}\text { Cervical cancer, breast } \\
\text { cancer and colorectal } \\
\text { cancer screening, } \\
\text { Chlamydia screening, } \\
\text { Cholesterol screening } \\
\text { (cardiac), LDL } \\
\text { cholesterol testing } \\
\text { (diabetes), eye exams } \\
\text { (diabetes), HbA1c } \\
\text { testing, nephropathy } \\
\text { screening }\end{array}$ & $\begin{array}{l}\text { Cholesterol } \\
\text { management, } \\
\text { HbA1c control, } \\
\text { LDL cholesterol } \\
\text { control, blood } \\
\text { pressure control }\end{array}$ & $9 / 4$ \\
\hline $\begin{array}{l}\text { Burgers } \\
\text { et } a p^{55}\end{array}$ & $\begin{array}{l}\text { Survey, } 8973 \\
\text { patients, Range }\end{array}$ & $\begin{array}{l}\text { Range of } \\
\text { settings }\end{array}$ & $\begin{array}{l}\text { Chronic lung, } \\
\text { mental health, } \\
\text { hypertension, } \\
\text { heart disease, } \\
\text { diabetes, } \\
\text { arthritis, cancer }\end{array}$ & $\mathrm{P}$ & $\begin{array}{l}\text { Coordination of care } \\
\text { and overall experience } \\
\text { (Commonwealth Fund } \\
\text { International Health } \\
\text { Policy Survey) }\end{array}$ & Death score & Death score & None & $1 / 0$ \\
\hline $\begin{array}{l}\text { Kaplan } \\
\text { et } a 2^{5}\end{array}$ & $\begin{array}{l}\text { Randomised } \\
\text { control trial, } 252 \\
\text { patients, USA }\end{array}$ & $\begin{array}{l}\text { Range of } \\
\text { settings }\end{array}$ & $\begin{array}{l}\text { Ulcer disease, } \\
\text { hypertension, } \\
\text { diabetes, breast } \\
\text { cancer }\end{array}$ & $\mathrm{P}$ & $\begin{array}{l}\text { Physician-patient } \\
\text { communication } \\
\text { (assessment of audio } \\
\text { tape and questionnaire) }\end{array}$ & $\begin{array}{l}\text { Physiological measures } \\
\text { taken at visit and } \\
\text { patients' self-rated } \\
\text { health status survey. }\end{array}$ & $\begin{array}{l}\text { Follow-up blood } \\
\text { glucose and blood } \\
\text { pressure, functional } \\
\text { health status, } \\
\text { self-reported health } \\
\text { status. }\end{array}$ & None & $4 / 0$ \\
\hline Jha et $a f^{3}$ & $\begin{array}{l}\text { Cross-sectional } \\
\text { study, } 2429 \\
\text { settings, USA }\end{array}$ & Hospital & $\begin{array}{l}\text { Acute } \\
\text { myocardial } \\
\text { infarction, } \\
\text { congestive heart } \\
\text { failure, } \\
\text { pneumonia } \\
\text { complications } \\
\text { from surgery }\end{array}$ & $\mathrm{O}$ & $\begin{array}{l}\text { Patient communication } \\
\text { with clinicians, } \\
\text { experience of nursing } \\
\text { services, discharge } \\
\text { planning (Hospital } \\
\text { Consumer Assessment } \\
\text { of Healthcare Providers } \\
\text { and Systems } \\
\text { (HCAHPS) survey) }\end{array}$ & $\begin{array}{l}\text { Technical quality of } \\
\text { care using Hospital } \\
\text { Quality Alliance (HQA) } \\
\text { score }\end{array}$ & $\begin{array}{l}\text { Technical quality of } \\
\text { care in AMI, congestive } \\
\text { heart failure (CHF), } \\
\text { pneumonia, surgical } \\
\text { care }\end{array}$ & None & $4 / 0$ \\
\hline
\end{tabular}




\begin{tabular}{|c|c|c|c|c|c|c|c|c|c|}
\hline Author & $\begin{array}{l}\text { Type of study, } \\
\text { sample size, } \\
\text { country }\end{array}$ & Setting & Disease focus & $\begin{array}{l}\text { Unit of } \\
\text { analysis } \\
\text { (patient } \\
\text { (P) or } \\
\text { org (O) }\end{array}$ & $\begin{array}{l}\text { Patient experience } \\
\text { focus and method } \\
\text { used }\end{array}$ & $\begin{array}{l}\text { Safety and } \\
\text { effectiveness } \\
\text { measure }\end{array}$ & $\begin{array}{l}\text { Association } \\
\text { demonstrated }\end{array}$ & $\begin{array}{l}\text { Association not } \\
\text { demonstrated }\end{array}$ & $\begin{array}{l}\text { Assoc. } \\
\text { Found } \\
\text { vs NOT } \\
\text { found }\end{array}$ \\
\hline Rao et $a f^{47}$ & $\begin{array}{l}\text { Cross-sectional } \\
\text { study, } 3487 \\
\text { patients, UK }\end{array}$ & Primary care & $\begin{array}{l}\text { Hypertension, } \\
\text { Influenza } \\
\text { vaccination }\end{array}$ & $\mathrm{P}$ & $\begin{array}{l}\text { Older patients' } \\
\text { experience of technical } \\
\text { quality of care (General } \\
\text { Practice Assessment } \\
\text { survey) }\end{array}$ & $\begin{array}{l}\text { Technical quality of } \\
\text { care-(medical } \\
\text { records) }\end{array}$ & None & $\begin{array}{l}\text { Hypertension } \\
\text { monitoring and } \\
\text { control, influenza } \\
\text { vaccination. }\end{array}$ & $0 / 3$ \\
\hline $\begin{array}{l}\text { Meterko } \\
\text { et } a F^{6}\end{array}$ & $\begin{array}{l}\text { Cohort study, } \\
1858 \text { patients, } \\
\text { USA }\end{array}$ & $\begin{array}{l}\text { Veteran } \\
\text { Affairs } \\
\text { Medical } \\
\text { Centres }\end{array}$ & $\begin{array}{l}\text { Acute } \\
\text { myocardial } \\
\text { infarction }\end{array}$ & $P$ & $\begin{array}{l}\text { Patient-centred care, } \\
\text { access, courtesy, } \\
\text { information, } \\
\text { coordination, patient } \\
\text { preferences, emotional } \\
\text { support, family } \\
\text { involvement, physical } \\
\text { comfort (VA Survey of } \\
\text { Healthcare } \\
\text { Experiences of Patients } \\
\text { (SHEP)) }\end{array}$ & $\begin{array}{l}\text { Survival 1-year } \\
\text { postdischarge }\end{array}$ & $\begin{array}{l}\text { Survival 1-year post } \\
\text { discharge }\end{array}$ & None & $1 / 0$ \\
\hline $\begin{array}{l}\text { Vincent } \\
\text { et } a^{5^{6}}\end{array}$ & $\begin{array}{l}\text { Cohort survey } \\
227 \text { patients, } \\
\text { UK }\end{array}$ & $\begin{array}{l}\text { Range of } \\
\text { settings }\end{array}$ & Varied & $P$ & $\begin{array}{l}\text { Accountability, } \\
\text { explanation, standards } \\
\text { of care, compensation } \\
\text { (questionnaire) }\end{array}$ & Legal action & Legal action & None & $1 / 0$ \\
\hline $\begin{array}{l}\text { Agoritsas } \\
\text { et } a \Gamma^{7}\end{array}$ & $\begin{array}{l}\text { Cohort patient } \\
\text { survey, } 1518 \\
\text { patients, } \\
\text { Switzerland }\end{array}$ & Hospital & Varied & $\mathrm{P}$ & $\begin{array}{l}\text { Global rating of care } \\
\text { and respect and dignity } \\
\text { questions (Picker } \\
\text { survey) }\end{array}$ & $\begin{array}{l}\text { Patient reports of } \\
\text { undesirable events } \\
\text { (survey) }\end{array}$ & $\begin{array}{l}\text { Neglect of important } \\
\text { information by } \\
\text { healthcare staff, pain } \\
\text { control, needless } \\
\text { repetition of a test, } \\
\text { being handled with } \\
\text { roughness }\end{array}$ & None & $4 / 0$ \\
\hline Flocke et $a{ }^{\beta 7}$ & $\begin{array}{l}\text { Cross-sectional } \\
\text { study, } 2889 \\
\text { patients, USA }\end{array}$ & Primary care & Varied & $\mathrm{P}$ & $\begin{array}{l}\text { Interpersonal } \\
\text { communication, } \\
\text { physician's knowledge } \\
\text { of patient, coordination } \\
\text { (Components of } \\
\text { Primary Care } \\
\text { Instrument }(\mathrm{CPCI}) \text { ) }\end{array}$ & $\begin{array}{l}\text { Use of preventive care } \\
\text { services (screening, } \\
\text { health habit counselling } \\
\text { services, immunisation } \\
\text { services) }\end{array}$ & $\begin{array}{l}\text { Screening, health habit } \\
\text { counselling, } \\
\text { immunisation }\end{array}$ & None & $3 / 0$ \\
\hline $\begin{array}{l}\text { Jackson, } \\
\text { J. et } a^{58}\end{array}$ & $\begin{array}{l}\text { Quantitative } \\
\text { cohort study } \\
500 \text { patients, } \\
\text { USA }\end{array}$ & $\begin{array}{l}\text { General } \\
\text { medicine } \\
\text { walk-in clinic }\end{array}$ & Varied & $P$ & $\begin{array}{l}\text { Patient satisfaction } \\
\text { (Research and } \\
\text { Development (RAND) } \\
\text { 9-item survey) }\end{array}$ & $\begin{array}{l}\text { Functional status } \\
\text { (Medical Outcomes } \\
\text { Study Short-Form } \\
\text { Health Survey (SF-6)), } \\
\text { symptom resolution, } \\
\text { (RAND 9-item survey), } \\
\text { follow-up visits }\end{array}$ & $\begin{array}{l}\text { Symptom resolution, } \\
\text { repeat visits, functional } \\
\text { status }\end{array}$ & None & $3 / 0$ \\
\hline
\end{tabular}




\begin{tabular}{|c|c|c|c|c|c|c|c|c|c|}
\hline Author & $\begin{array}{l}\text { Type of study, } \\
\text { sample size, } \\
\text { country }\end{array}$ & Setting & Disease focus & $\begin{array}{l}\text { Unit of } \\
\text { analysis } \\
\text { (patient } \\
\text { (P) or } \\
\text { org (O) }\end{array}$ & $\begin{array}{l}\text { Patient experience } \\
\text { focus and method } \\
\text { used }\end{array}$ & $\begin{array}{l}\text { Safety and } \\
\text { effectiveness } \\
\text { measure }\end{array}$ & $\begin{array}{l}\text { Association } \\
\text { demonstrated }\end{array}$ & $\begin{array}{l}\text { Association not } \\
\text { demonstrated }\end{array}$ & $\begin{array}{l}\text { Assoc. } \\
\text { Found } \\
\text { vs NOT } \\
\text { found }\end{array}$ \\
\hline Clark et $a{ }^{41}$ & $\begin{array}{l}\text { Randomised } \\
\text { control trial } 731 \\
\text { patients, USA }\end{array}$ & $\begin{array}{l}\text { Range of } \\
\text { settings }\end{array}$ & Asthma & $P$ & $\begin{array}{l}\text { Patient experience of } \\
\text { physician } \\
\text { communication (patient } \\
\text { interviews and Likert } \\
\text { scale) }\end{array}$ & $\begin{array}{l}\text { Emergency department } \\
\text { visits, hospitalisations, } \\
\text { office phone calls and } \\
\text { visits, urgent office } \\
\text { visits (survey+medical } \\
\text { chart review of } 6 \% \text { of } \\
\text { patients to verify } \\
\text { responses) }\end{array}$ & $\begin{array}{l}\text { Number of office visits, } \\
\text { emergency visits, } \\
\text { urgent office visits, } \\
\text { phone calls, } \\
\text { hospitalisations }\end{array}$ & None & $5 / 0$ \\
\hline Raiz et $a^{R o}$ & $\begin{array}{l}\text { Quantitative } \\
\text { cohort study, } \\
357 \text { patients, } \\
\text { USA }\end{array}$ & Primary care & Renal transplant & $P$ & $\begin{array}{l}\text { Patient faith in doctor } \\
\text { (Multidimensional } \\
\text { Health Locus of Control } \\
\text { Scale (MHLC)) }\end{array}$ & Medication compliance & $\begin{array}{l}\text { Remembering } \\
\text { medications, taking } \\
\text { medications as } \\
\text { prescribed }\end{array}$ & None & $2 / 0$ \\
\hline Kahn et $a^{\beta 2}$ & $\begin{array}{l}\text { Cohort study, } \\
881 \text { patients, } \\
\text { USA }\end{array}$ & Hospitals & Breast cancer & $\mathrm{P}$ & $\begin{array}{l}\text { Level of physician } \\
\text { support, participation in } \\
\text { decision-making and } \\
\text { information on side } \\
\text { effects (survey) }\end{array}$ & Medication adherence & Ongoing tamoxifen use & None & $1 / 0$ \\
\hline $\begin{array}{l}\text { Plomondon } \\
\text { et } a^{R^{2}}\end{array}$ & $\begin{array}{l}\text { Cohort study, } \\
1815 \text { patients, } \\
\text { USA }\end{array}$ & Hospital & $\begin{array}{l}\text { Myocardial } \\
\text { infarction }\end{array}$ & $P$ & $\begin{array}{l}\text { Satisfaction with } \\
\text { explanations from their } \\
\text { doctor, overall } \\
\text { satisfaction with } \\
\text { treatment (Seattle } \\
\text { Angina questionnaire) }\end{array}$ & $\begin{array}{l}\text { Presence of angina } \\
\text { (Seattle Angina } \\
\text { Questionnaire) }\end{array}$ & Presence of angina & None & $1 / 0$ \\
\hline $\begin{array}{l}\text { Fuertes } \\
\text { et } a l^{19}\end{array}$ & $\begin{array}{l}\text { Survey, } 152 \\
\text { patients, USA }\end{array}$ & Hospital & Neurology & $P$ & $\begin{array}{l}\text { Physician-patient } \\
\text { communication, } \\
\text { physician-patient } \\
\text { working alliance, } \\
\text { empathy, multicultural } \\
\text { competence } \\
\text { (questionnaire) }\end{array}$ & $\begin{array}{l}\text { Adherence to medical } \\
\text { treatment (adherence } \\
\text { Self-Efficacy Scale and } \\
\text { Medical Outcome } \\
\text { Study (MOS) } \\
\text { adherence scale) }\end{array}$ & Adherence to treatment & None & $1 / 0$ \\
\hline Lewis et $a \beta^{31}$ & $\begin{array}{l}\text { Qualitative } \\
\text { cohort study, } \\
191 \text { patients, } \\
\text { USA }\end{array}$ & Primary care & Pain & $P$ & $\begin{array}{l}\text { Doctor-patient } \\
\text { communication (survey) }\end{array}$ & $\begin{array}{l}\text { Medication adherence } \\
\text { (Prescription Drug Use } \\
\text { Questionnaire (PDUQ)) }\end{array}$ & $\begin{array}{l}\text { Use of prescribed } \\
\text { opioid medications }\end{array}$ & None & $1 / 0$ \\
\hline Safran et $a^{59}$ & $\begin{array}{l}\text { Cross-sectional } \\
\text { study, } 7204 \\
\text { patients, USA }\end{array}$ & Primary care & Varied & $P$ & $\begin{array}{l}\text { Accessibility, continuity, } \\
\text { integration, clinical } \\
\text { interaction, } \\
\text { interpersonal aspects, } \\
\text { trust (The Primary Care } \\
\text { Assessment Survey) }\end{array}$ & $\begin{array}{l}\text { Adherence to } \\
\text { physician's advice, } \\
\text { health status, health } \\
\text { outcomes (Medical } \\
\text { Outcomes Study } \\
\text { (MOS), Behavioural risk } \\
\text { factor survey) }\end{array}$ & $\begin{array}{l}\text { Adherence, health } \\
\text { status }\end{array}$ & Health outcomes & $2 / 1$ \\
\hline
\end{tabular}




\begin{tabular}{|c|c|c|c|c|c|c|c|c|c|}
\hline Author & $\begin{array}{l}\text { Type of study, } \\
\text { sample size, } \\
\text { country }\end{array}$ & Setting & Disease focus & $\begin{array}{l}\text { Unit of } \\
\text { analysis } \\
\text { (patient } \\
\text { (P) or } \\
\text { org (O) }\end{array}$ & $\begin{array}{l}\text { Patient experience } \\
\text { focus and method } \\
\text { used }\end{array}$ & $\begin{array}{l}\text { Safety and } \\
\text { effectiveness } \\
\text { measure }\end{array}$ & $\begin{array}{l}\text { Association } \\
\text { demonstrated }\end{array}$ & $\begin{array}{l}\text { Association not } \\
\text { demonstrated }\end{array}$ & $\begin{array}{l}\text { Assoc. } \\
\text { Found } \\
\text { vs NOT } \\
\text { found }\end{array}$ \\
\hline Alamo et a ${ }^{60}$ & $\begin{array}{l}\text { Randomised } \\
\text { study, 81, } \\
\text { Spain }\end{array}$ & Primary care & $\begin{array}{l}\text { Chronic } \\
\text { musculoskeletal } \\
\text { pain (CMP), } \\
\text { fibromyalgia }\end{array}$ & $\mathrm{P}$ & $\begin{array}{l}\text { Patient-centreed-care } \\
\text { ('Gatha-Res } \\
\text { questionnaire' and } \\
\text { follow-up phone call) }\end{array}$ & $\begin{array}{l}\text { Pain (Visual Analogue } \\
\text { Scale (VAS) anxiety } \\
\text { (Oldberg scale of } \\
\text { anxiety and depression } \\
(\text { GHQ)) }\end{array}$ & $\begin{array}{l}\text { Anxiety, number of } \\
\text { tender points (pain) }\end{array}$ & $\begin{array}{l}\text { Pain, pain } \\
\text { intensity, pain as } \\
\text { a problem, } \\
\text { number of } \\
\text { associated } \\
\text { symptoms, } \\
\text { depression, } \\
\text { physical mobility, } \\
\text { social isolation, } \\
\text { emotional } \\
\text { reaction, sleep }\end{array}$ & $2 / 10$ \\
\hline Fan et $a^{\beta 1}$ & $\begin{array}{l}\text { Survey, } 21689 \\
\text { patients, USA }\end{array}$ & Primary care & $\begin{array}{l}\text { Cardiac care, } \\
\text { diabetes, } \\
\text { congestive } \\
\text { obstructive } \\
\text { pulmonary } \\
\text { disorder } \\
\text { (COPD) }\end{array}$ & $P$ & $\begin{array}{l}\text { Communication skills } \\
\text { and humanistic } \\
\text { qualities of primary } \\
\text { care physician (Seattle } \\
\text { Outpatient Satisfaction } \\
\text { Survey) }\end{array}$ & $\begin{array}{l}\text { Physical and emotional } \\
\text { aspects, coping ability } \\
\text { and symptom burden } \\
\text { for angina, COPD and } \\
\text { diabetes (Seattle } \\
\text { Angina Questionnaire } \\
\text { (SAQ), Obstructive } \\
\text { Lung Disease } \\
\text { Questionnaire } \\
\text { (SOLDQ), Diabetes } \\
\text { Questionnaire (SDQ)) }\end{array}$ & $\begin{array}{l}\text { Patient ability to deal } \\
\text { with all } 3 \text { diseases, } \\
\text { education for diabetes } \\
\text { patients, angina } \\
\text { stability, physical } \\
\text { limitation due to angina }\end{array}$ & $\begin{array}{l}\text { Self-reported } \\
\text { physical limitation } \\
\text { for angina and } \\
\text { COPD, symptom } \\
\text { burden for } \\
\text { diabetes, } \\
\text { complications for } \\
\text { diabetes }\end{array}$ & $7 / 4$ \\
\hline $\begin{array}{l}\text { O'Malley } \\
\text { et } a^{\beta 8}\end{array}$ & $\begin{array}{l}\text { Cross-sectional } \\
\text { study, } 961 \\
\text { patients, USA }\end{array}$ & Primary care & Varied & $P$ & Patient trust (survey) & $\begin{array}{l}\text { Use of preventive care } \\
\text { services }\end{array}$ & $\begin{array}{l}\text { Blood pressure } \\
\text { measurement, height } \\
\text { and weight } \\
\text { measurement, } \\
\text { cholesterol check, } \\
\text { papanicolaou test (pap) } \\
\text { tests, breast cancer } \\
\text { screening, colorectal } \\
\text { cancer screening, } \\
\text { discussion of diet, } \\
\text { discussion on } \\
\text { depression }\end{array}$ & None & $8 / 0$ \\
\hline Little et $a^{62}$ & $\begin{array}{l}\text { Survey, } 865 \\
\text { patients, UK }\end{array}$ & Primary care & varied & $P$ & $\begin{array}{l}\text { Patient centredness } \\
\text { (Survey) }\end{array}$ & $\begin{array}{l}\text { Enablement, symptom } \\
\text { burden, resource use }\end{array}$ & $\begin{array}{l}\text { Enablement, symptom } \\
\text { burden, referrals }\end{array}$ & $\begin{array}{l}\text { Re-attendance, } \\
\text { investigations }\end{array}$ & $3 / 2$ \\
\hline $\begin{array}{l}\text { Levinson } \\
\text { et } a f^{63}\end{array}$ & $\begin{array}{l}\text { Qualitative } \\
\text { cohort study, } \\
124 \text { physicians, } \\
\text { USA }\end{array}$ & Primary care & Varied & $P$ & $\begin{array}{l}\text { Physician-patient } \\
\text { communication } \\
\text { (assessment of } \\
\text { audiotape) }\end{array}$ & Malpractice & Malpractice claims & None & $1 / 0$ \\
\hline
\end{tabular}




\begin{tabular}{|c|c|c|c|c|c|c|c|c|c|}
\hline Author & $\begin{array}{l}\text { Type of study, } \\
\text { sample size, } \\
\text { country }\end{array}$ & Setting & Disease focus & $\begin{array}{l}\text { Unit of } \\
\text { analysis } \\
\text { (patient } \\
\text { (P) or } \\
\text { org (O) }\end{array}$ & $\begin{array}{l}\text { Patient experience } \\
\text { focus and method } \\
\text { used }\end{array}$ & $\begin{array}{l}\text { Safety and } \\
\text { effectiveness } \\
\text { measure }\end{array}$ & $\begin{array}{l}\text { Association } \\
\text { demonstrated }\end{array}$ & $\begin{array}{l}\text { Association not } \\
\text { demonstrated }\end{array}$ & $\begin{array}{l}\text { Assoc. } \\
\text { Found } \\
\text { vs NOT } \\
\text { found }\end{array}$ \\
\hline $\begin{array}{l}\text { Carcaise- } \\
\text { Edinboro and } \\
\text { Bradley }^{39}\end{array}$ & $\begin{array}{l}\text { Cross sectional } \\
\text { study, } 8488 \\
\text { patients, USA }\end{array}$ & Primary care & $\begin{array}{l}\text { Colorectal } \\
\text { cancer }\end{array}$ & $\mathrm{P}$ & $\begin{array}{l}\text { Patient-provider } \\
\text { communication } \\
\text { (Consumer } \\
\text { Assessment of } \\
\text { Healthcare Providers } \\
\text { and Systems (CAHPS) } \\
\text { survey) }\end{array}$ & $\begin{array}{l}\text { Colorectal Cancer } \\
\text { screening, fecal occult } \\
\text { blood testing and } \\
\text { colonoscopy (Medical } \\
\text { Expenditure Panel } \\
\text { Survey) }\end{array}$ & $\begin{array}{l}\text { CRC screening, fecal } \\
\text { occult blood testing, } \\
\text { colonoscopy }\end{array}$ & None & $3 / 0$ \\
\hline $\begin{array}{l}\text { Schneider } \\
\text { et } a^{\beta 3}\end{array}$ & $\begin{array}{l}\text { Cross-sectional } \\
\text { analysis study, } \\
554 \text { patients, } \\
\text { USA }\end{array}$ & Primary care & HIV & $\mathrm{P}$ & $\begin{array}{l}\text { Physician-patient } \\
\text { relationship (survey) }\end{array}$ & Adherence (survey) & $\begin{array}{l}\text { Adherence to } \\
\text { antiretroviral therapy }\end{array}$ & None & $1 / 0$ \\
\hline $\begin{array}{l}\text { Schoenthaler } \\
\text { et } a^{\beta 4}\end{array}$ & $\begin{array}{l}\text { Cross-sectional } \\
\text { study, } 439 \\
\text { patients, USA }\end{array}$ & Primary care & Hypertension & $\mathrm{P}$ & $\begin{array}{l}\text { Patients' perceptions of } \\
\text { providers' } \\
\text { communication (survey) }\end{array}$ & $\begin{array}{l}\text { Medication adherence } \\
\text { (Morisky self-report } \\
\text { measure) }\end{array}$ & Medication adherence & None & $1 / 0$ \\
\hline $\begin{array}{l}\text { Slatore } \\
\text { et } a^{64}\end{array}$ & $\begin{array}{l}\text { Cross-sectional } \\
\text { study, } 342 \\
\text { patients, USA }\end{array}$ & $\begin{array}{l}\text { Range of } \\
\text { settings }\end{array}$ & COPD & $\mathrm{P}$ & $\begin{array}{l}\text { Patient-clinician } \\
\text { communication (Quality } \\
\text { of communication } \\
\text { questionnaire (QOC)) }\end{array}$ & $\begin{array}{l}\text { Self-reported breathing } \\
\text { problem confidence } \\
\text { and general self-rated } \\
\text { health (survey) }\end{array}$ & $\begin{array}{l}\text { Confidence in dealing } \\
\text { with breathing problems }\end{array}$ & Self-rated health & $1 / 1$ \\
\hline $\begin{array}{l}\text { Lee and } \\
\operatorname{Lin}^{65}\end{array}$ & $\begin{array}{l}\text { Cohort study, } \\
480 \text { patients, } \\
\text { Taiwan }\end{array}$ & $\begin{array}{l}\text { Range of } \\
\text { settings }\end{array}$ & Type 2 diabetes & $\mathrm{P}$ & $\begin{array}{l}\text { Trust in physicians } \\
\text { (survey) }\end{array}$ & $\begin{array}{l}\text { Self-efficacy, } \\
\text { adherence, health } \\
\text { outcomes } \\
\text { (Multidimensional } \\
\text { Diabetes Questionnaire } \\
\text { and 12-Item } \\
\text { Short-Form Health } \\
\text { survey (SF-12)) }\end{array}$ & $\begin{array}{l}\text { Physical HRQoL, } \\
\text { mental HRQoL, body } \\
\text { mass index HbA1c, } \\
\text { triglycerides, } \\
\text { complications, } \\
\text { self-efficacy, outcome } \\
\text { expectations, } \\
\text { adherence }\end{array}$ & None & $9 / 0$ \\
\hline $\begin{array}{l}\text { Heisler } \\
\text { et } a^{\beta 5}\end{array}$ & $\begin{array}{l}\text { Survey, } 1314 \\
\text { patients, USA }\end{array}$ & Primary care & Diabetes & $\mathrm{P}$ & $\begin{array}{l}\text { Physician } \\
\text { communication, } \\
\text { physician interaction } \\
\text { styles, participatory } \\
\text { decision-making } \\
\text { (Questionnaire) }\end{array}$ & $\begin{array}{l}\text { Disease management } \\
\text { (surveys and national } \\
\text { databases) }\end{array}$ & $\begin{array}{l}\text { Overall } \\
\text { self-management, } \\
\text { diabetes diet, } \\
\text { medication compliance, } \\
\text { exercise, blood glucose } \\
\text { monitoring, foot care. }\end{array}$ & Exercise & $6 / 1$ \\
\hline \multirow[t]{2}{*}{$\begin{array}{l}\text { Lee and } \\
\operatorname{Lin}^{66}\end{array}$} & $\begin{array}{l}\text { Cohort study, } \\
614 \text { patients, } \\
\text { Taiwan }\end{array}$ & $\begin{array}{l}\text { Range of } \\
\text { settings }\end{array}$ & Type 2 diabetes & $\mathrm{P}$ & $\begin{array}{l}\text { Patients' perceptions of } \\
\text { support, autonomy, } \\
\text { trust, satisfaction } \\
\text { (Healthcare Climate } \\
\text { Questionnaire and } \\
\text { Autonomy Preference } \\
\text { Index (API)) }\end{array}$ & $\begin{array}{l}\text { Glycosylated } \\
\text { haemoglobin (HbA1C) } \\
\text { (medical records) } \\
\text { Physical and mental } \\
\text { health-related quality of } \\
\text { life (HRQoL) (SF-12) }\end{array}$ & $\begin{array}{l}\text { Physical HRQoL, } \\
\text { mental HRQoL }\end{array}$ & $\begin{array}{l}\text { Information } \\
\text { preference } \\
\text { interaction, } \\
\text { HbA1C }\end{array}$ & $2 / 2$ \\
\hline & & & & & & & & & Continued \\
\hline
\end{tabular}




\begin{tabular}{|c|c|c|c|c|c|c|c|c|c|}
\hline Author & $\begin{array}{l}\text { Type of study, } \\
\text { sample size, } \\
\text { country }\end{array}$ & Setting & Disease focus & $\begin{array}{l}\text { Unit of } \\
\text { analysis } \\
\text { (patient } \\
\text { (P) or } \\
\text { org (O) }\end{array}$ & $\begin{array}{l}\text { Patient experience } \\
\text { focus and method } \\
\text { used }\end{array}$ & $\begin{array}{l}\text { Safety and } \\
\text { effectiveness } \\
\text { measure }\end{array}$ & $\begin{array}{l}\text { Association } \\
\text { demonstrated }\end{array}$ & $\begin{array}{l}\text { Association not } \\
\text { demonstrated }\end{array}$ & $\begin{array}{l}\text { Assoc. } \\
\text { Found } \\
\text { vs NOT } \\
\text { found }\end{array}$ \\
\hline $\begin{array}{l}\text { Kennedy } \\
\text { A. et } a \rho^{7}\end{array}$ & $\begin{array}{l}\text { Randomised } \\
\text { control trial, } 700 \\
\text { patients, UK }\end{array}$ & Hospital & $\begin{array}{l}\text { Inflammatory } \\
\text { bowel Disease }\end{array}$ & $P$ & $\begin{array}{l}\text { Patient-centred-care } \\
\text { (interviews) }\end{array}$ & $\begin{array}{l}\text { Resource use, } \\
\text { self-rated physical and } \\
\text { mental health, } \\
\text { enablement (patient } \\
\text { diaries, questionnaires, } \\
\text { medical records) }\end{array}$ & $\begin{array}{l}\text { Ability to cope with } \\
\text { condition, symptom } \\
\text { relapses, hospital visits, } \\
\text { appointments made }\end{array}$ & $\begin{array}{l}\text { Physical } \\
\text { functioning, role } \\
\text { limitations, social } \\
\text { functioning, } \\
\text { mental health, } \\
\text { energy/vitality, } \\
\text { pain, general } \\
\text { health perception, } \\
\text { anxiety, number } \\
\text { of relapses, } \\
\text { number of } \\
\text { medically-defined } \\
\text { relapses, average } \\
\text { relapse duration, } \\
\text { frequency of GP } \\
\text { visits, delay } \\
\text { before starting } \\
\text { treatment }\end{array}$ & $4 / 13$ \\
\hline $\begin{array}{l}\text { Stewart } \\
\text { et } a l^{42}\end{array}$ & $\begin{array}{l}\text { Observational } \\
\text { cohort study, } \\
315 \text { patients, } \\
\text { Canada }\end{array}$ & Primary care & General & $\mathrm{P}$ & $\begin{array}{l}\text { Patient-centred } \\
\text { communication } \\
\text { (assessment of } \\
\text { audiotape and } \\
\text { Patient-Centred } \\
\text { Communication Score } \\
\text { tool) }\end{array}$ & $\begin{array}{l}\text { Discomfort (VAS) } \\
\text { symptom severity } \\
\text { severity (Visual } \\
\text { Analogue Scale), } \\
\text { Health Status (Short } \\
\text { Form-36 SF-36) Quality } \\
\text { of care provision (chart } \\
\text { review by doctors) }\end{array}$ & $\begin{array}{l}\text { Symptom discomfort } \\
\text { and concern, } \\
\text { self-reported health, } \\
\text { diagnostic tests, } \\
\text { referrals and visits to } \\
\text { the family physician }\end{array}$ & None & $5 / 2$ \\
\hline $\begin{array}{l}\text { Kinnersley } \\
\text { et } a^{68}\end{array}$ & $\begin{array}{l}\text { Observational } \\
\text { study, } 143 \\
\text { patients, UK }\end{array}$ & Primary care & Varied & $P$ & $\begin{array}{l}\text { Patient-centredness } \\
\text { (assessment of } \\
\text { audiotape and } \\
\text { questionnaires) }\end{array}$ & $\begin{array}{l}\text { Symptom resolution, } \\
\text { resolution of concerns, } \\
\text { functional health status } \\
\text { (Questionnaire) }\end{array}$ & None & $\begin{array}{l}\text { Resolution of } \\
\text { symptoms, } \\
\text { resolution of } \\
\text { concerns, } \\
\text { functional health } \\
\text { status }\end{array}$ & $0 / 3$ \\
\hline $\begin{array}{l}\text { Solberg } \\
\text { et } a^{F^{1}}\end{array}$ & $\begin{array}{l}\text { Survey, } 3109 \\
\text { patients, USA }\end{array}$ & $\begin{array}{l}\text { Primary care } \\
\text { - } \\
\text { multispecialty } \\
\text { group }\end{array}$ & Varied & $P$ & $\begin{array}{l}\text { Patient experience of } \\
\text { errors (survey) }\end{array}$ & $\begin{array}{l}\text { Review of errors (chart } \\
\text { audits and physician } \\
\text { reviewer judgements) }\end{array}$ & None & None & $1 / 0$ \\
\hline Isaac et $a{ }^{\rho}$ & $\begin{array}{l}\text { Cross-sectional } \\
\text { study, } 927 \\
\text { hospitals, USA }\end{array}$ & Hospital & $\begin{array}{l}\text { Acute } \\
\text { myocardial } \\
\text { infarction, }\end{array}$ & $\mathrm{O}$ & $\begin{array}{l}\text { General patient } \\
\text { experiences (Hospital } \\
\text { Consumer Assessment }\end{array}$ & $\begin{array}{l}\text { Processes of care } \\
\text { (Health Quality Alliance }\end{array}$ & $\begin{array}{l}\text { Decubitus ulcer rates, } \\
\text { infections, processes of } \\
\text { care for pneumonia, }\end{array}$ & Failure to rescue & $11 / 1$ \\
\hline
\end{tabular}


Table 5 Continued

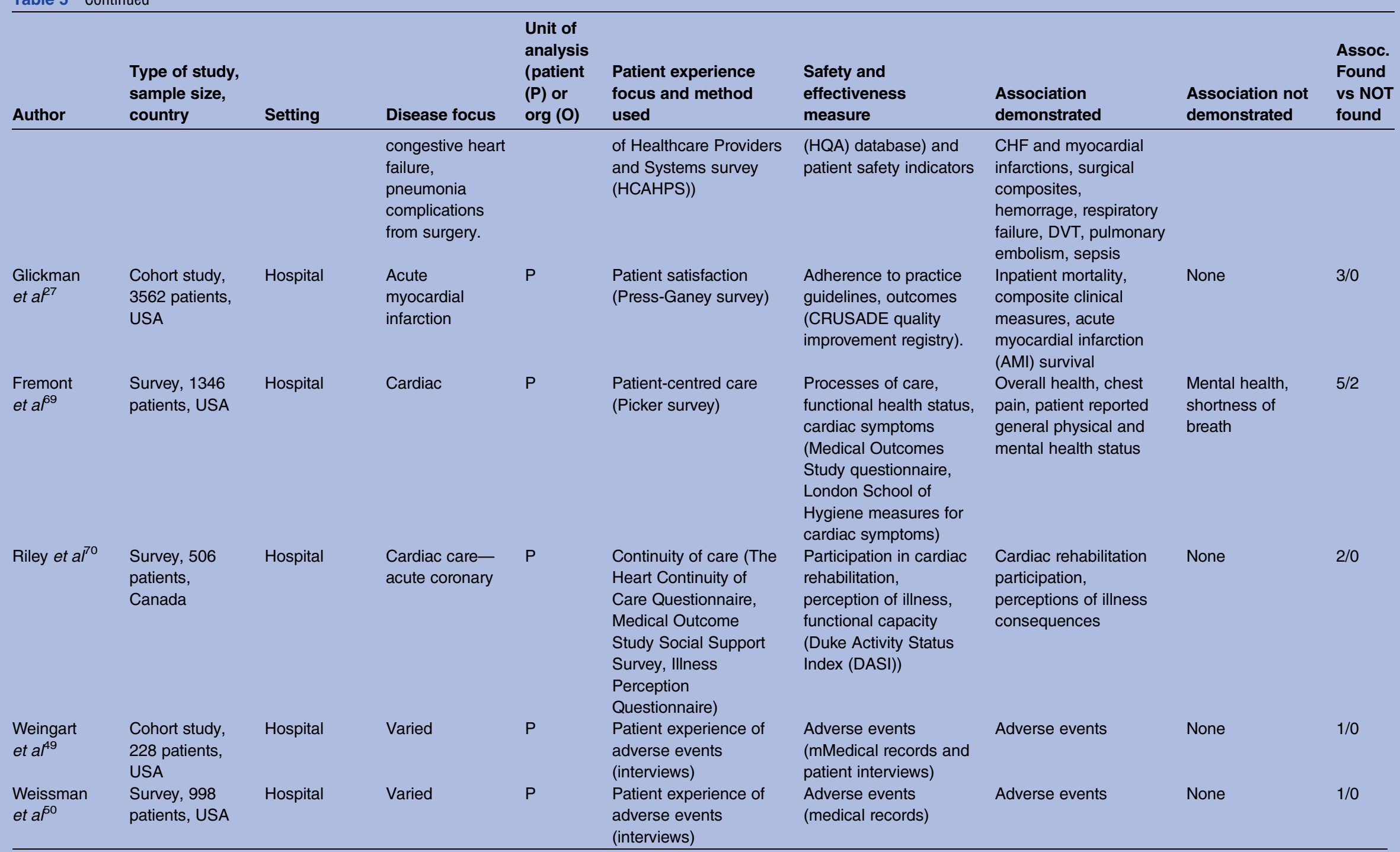

HRQoL, health-related quality of life. 


\begin{tabular}{|c|c|c|c|c|c|c|c|c|}
\hline Authors & $\begin{array}{l}\text { Time span and } \\
\text { studies } \\
\text { meeting } \\
\text { inclusion } \\
\text { criteria } \\
\end{array}$ & $\begin{array}{l}\text { Healthcare } \\
\text { setting }\end{array}$ & $\begin{array}{l}\text { Disease areas } \\
\text { covered }\end{array}$ & $\begin{array}{l}\text { Unit of } \\
\text { analysis }\end{array}$ & $\begin{array}{l}\text { Patient experience } \\
\text { focus (and } \\
\text { measurement } \\
\text { methods) }\end{array}$ & $\begin{array}{l}\text { Safety and effectiveness } \\
\text { measure-association } \\
\text { demonstrated - }\end{array}$ & $\begin{array}{l}\text { Safety and effectiveness } \\
\text { measure-association not } \\
\text { demonstrated }\end{array}$ & $\begin{array}{l}\text { Assocs } \\
\text { found vs } \\
\text { not } \\
\text { found } \\
\end{array}$ \\
\hline Blasi et $a l^{11}$ & $\begin{array}{l}1974-1998, \\
4 \text { of } 25\end{array}$ & $\begin{array}{l}\text { Range of } \\
\text { settings }\end{array}$ & $\begin{array}{l}\text { Asthma, hypertension, } \\
\text { cancer, insomnia, } \\
\text { menopause, obesity, } \\
\text { tonsillitis }\end{array}$ & $\mathrm{P}$ & $\begin{array}{l}\text { Provider behaviour } \\
\text { and communication } \\
\text { (grading of } \\
\text { consultations) }\end{array}$ & $\begin{array}{l}\text { Health status, symptom } \\
\text { improvement, treatment } \\
\text { effectiveness, fear of injection, } \\
\text { anxiety, ratings of pain, number } \\
\text { of doctor visits, pain, speed of } \\
\text { recovery }\end{array}$ & $\begin{array}{l}\text { Comfort, recovery time, return } \\
\text { visits }\end{array}$ & $9 / 3$ \\
\hline Drotar $^{29}$ & $\begin{array}{l}1998-2008, \\
4 \text { of } 22\end{array}$ & $\begin{array}{l}\text { Range of } \\
\text { settings }\end{array}$ & $\begin{array}{l}\text { Asthma, cystic } \\
\text { fibrosis, diabetes, } \\
\text { epilepsy, inflammatory } \\
\text { bowel disease, } \\
\text { juvenile rheumatoid } \\
\text { arthritis }\end{array}$ & $\mathrm{P}$ & $\begin{array}{l}\text { Physician and staff } \\
\text { behaviour (surveys, } \\
\text { interviews, medical } \\
\text { records) }\end{array}$ & $\begin{array}{l}\text { Treatment adherence, } \\
\text { compliance, office visits, phone } \\
\text { calls, hospitalisations }\end{array}$ & Medication adherence & $5 / 1$ \\
\hline Hall et $a l^{72}$ & $\begin{array}{l}1990-2009, \\
10 \text { of } 14\end{array}$ & $\begin{array}{l}\text { Range of } \\
\text { settings }\end{array}$ & $\begin{array}{l}\text { Brain injury, } \\
\text { musculoskeletal } \\
\text { conditions, cardiac } \\
\text { conditions, trauma, } \\
\text { back, neck and } \\
\text { shoulder pain }\end{array}$ & $\mathrm{P}$ & $\begin{array}{l}\text { Therapist-patient } \\
\text { relationship, } \\
\text { therapeutic alliance } \\
\text { (surveys, audio/video } \\
\text { taped session) }\end{array}$ & $\begin{array}{l}\text { Adherence, employment status, } \\
\text { physical training, therapeutic } \\
\text { success, perceived effect of } \\
\text { treatment, pain, physical } \\
\text { function, depression, general } \\
\text { health status, attendance, } \\
\text { floor-bench lifts, global } \\
\text { assessment scores, ability to } \\
\text { perform activities of daily living } \\
\text { (ADLs), mobility }\end{array}$ & $\begin{array}{l}\text { Weekly physical training, } \\
\text { disability, productivity, } \\
\text { depression, functional status, } \\
\text { adherence }\end{array}$ & $18 / 6$ \\
\hline $\begin{array}{l}\text { Stevenson } \\
\text { et } a l^{73}\end{array}$ & $\begin{array}{l}1991-2000 \\
7 \text { of } 134\end{array}$ & $\begin{array}{l}\text { Range of } \\
\text { settings }\end{array}$ & $\begin{array}{l}\text { Hypertension, asthma, } \\
\text { chronic obstructive } \\
\text { pulmonary disorder, } \\
\text { ovarian cancer, } \\
\text { epilepsy, } \\
\text { hyperlipidaemia }\end{array}$ & $\mathrm{P}$ & $\begin{array}{l}\text { Doctor-patient } \\
\text { communication } \\
\text { (surveys) }\end{array}$ & $\begin{array}{l}\text { Self-reported adherence, blood } \\
\text { pressure control, general } \\
\text { physician practice visits, } \\
\text { hospitalisations, emergency } \\
\text { room visits for children with } \\
\text { asthma, quality of life for COPD } \\
\text { patients, oral contraceptive } \\
\text { adherence, adherence to } \\
\text { antiepileptic drugs, pain control } \\
\text { following gynaecological } \\
\text { surgery, adherence to } \\
\text { medication for depression }\end{array}$ & $\begin{array}{l}\text { Length of visits to doctor for } \\
\text { asthma patients, health status } \\
\text { and use of healthcare services } \\
\text { for epilepsy patients, } \\
\text { adherence to Niacin and bile } \\
\text { acid sequestrant therapy }\end{array}$ & $9 / 5$ \\
\hline $\begin{array}{l}\text { Saultz and } \\
\text { Lochner }^{44}\end{array}$ & $\begin{array}{l}\text { 1967-2002, } \\
41 \text { studies }\end{array}$ & $\begin{array}{l}\text { Range of } \\
\text { settings }\end{array}$ & Varied & $\mathrm{P}$ & $\begin{array}{l}\text { Continuity of care - } \\
\text { ongoing relationship } \\
\text { between individual } \\
\text { doctor and patient }\end{array}$ & $\begin{array}{l}\text { Hospitalisation rate, hospital } \\
\text { readmission, length of stay, } \\
\text { influenza immunisation, } \\
\text { preventive care, antibiotic } \\
\text { compliance, intensive care unit }\end{array}$ & $\begin{array}{l}\text { Diabetes (HbA1C, lipid } \\
\text { control, blood pressure control, } \\
\text { presence of diabetic } \\
\text { complications), blood glucose } \\
\text { control, functional ability of }\end{array}$ & $51 / 30$ \\
\hline
\end{tabular}

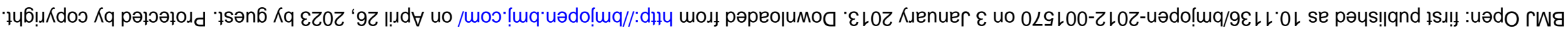


Time span and

studies

meeting

inclusion

Authors

criteria

sealthcare

Disease areas

covered

\section{Hall, Roter and Meta-analysis}

Katz $^{74}$

41 studies

Range of Varied

settings

Varied

$\begin{array}{llll}\begin{array}{l}\text { Sans-Coralles } \\ \text { et } a f^{43}\end{array} & \begin{array}{l}1984-2005, \\ 9 \text { of } 20\end{array} & \begin{array}{l}\text { Primary } \\ \text { care }\end{array} & \begin{array}{l}\text { No specific disease } \\ \text { focus }\end{array}\end{array}$

Hsiao and

Boult $^{45}$

1984-2003

3 of 14
Primary

care
No specific disease $\quad P$ focus
Patient experience

focus (and

measurement

methods)

(surveys, continuity

of care index)

Clinician-patient

communication

(surveys, interviews,

observations,

assessment of video

or audio)

Trust in physician,

Patient-physician

agreement,

adequacy information

(surveys)

\section{Safety and effectiveness}

measure-association

demonstrated -

days, Neonatal morbidity, Apgar score, Birth weight, rates and timeliness of childhood

immunisations, health-related quality of life, recommended diabetes care measures,

glucose control, PAP tests,

mammogram rate, breast

exams, surgical operation rates

hypertension control, presence

of depression, relationship

problems, adverse events in

hospitalsed patients, degree of patient enablement, rheumatic

fever incidence

Compliance (with 4 variables of $P E$ ), recall/understanding (with

4 variables of $P E$ )

Adherence to treatment

Compliance

Continuity of care, coordination of care, consultation time,

doctor-patient

relationship

(validated tools in

these different

domains)

Continuity with

physician (surveys

interviews, medical

Safety and effectiveness measure-association not demonstrated

Assocs

found vs not

Iderly patients, compliance with antibiotic therapy, well-child visits, blood

pressure checks in women, pregnancy complications, newborn mortality,

immunization rates, $\mathrm{NICU}$ admissions, Apgar scores, caesarean rate, length of

labour, indications for tonsillectomy

Compliance (with 1 variable of $8 / 2$

$\mathrm{PE}$ ), recall/understanding (with

1 variable of $P E$ )

Hospital admissions, length of stay, compliance, recovery from discomfort, emotional health, diagnostic tests, referrals, quality of care for asthma, diabetes and angina, symptom burden, receipt of preventive services

Hospitalisations for all

conditions and ambulatory

care-sensitive conditions, odds of hospitalisation(2), healthcare
Acute ambulatory

$21 / 15$

care-sensitive conditions,

mobility, pain, emotion,

activities of daily living, 


\begin{tabular}{|c|c|c|c|c|c|c|c|c|}
\hline Authors & $\begin{array}{l}\text { Time span and } \\
\text { studies } \\
\text { meeting } \\
\text { inclusion } \\
\text { criteria }\end{array}$ & $\begin{array}{l}\text { Healthcare } \\
\text { setting }\end{array}$ & $\begin{array}{l}\text { Disease areas } \\
\text { covered }\end{array}$ & $\begin{array}{l}\text { Unit of } \\
\text { analysis }\end{array}$ & $\begin{array}{l}\text { Patient experience } \\
\text { focus (and } \\
\text { measurement } \\
\text { methods) }\end{array}$ & $\begin{array}{l}\text { Safety and effectiveness } \\
\text { measure-association } \\
\text { demonstrated - }\end{array}$ & $\begin{array}{l}\text { Safety and effectiveness } \\
\text { measure-association not } \\
\text { demonstrated }\end{array}$ & $\begin{array}{l}\text { Assocs } \\
\text { found vs } \\
\text { not } \\
\text { found }\end{array}$ \\
\hline & & & & & $\begin{array}{l}\text { records, chart } \\
\text { reviews) }\end{array}$ & $\begin{array}{l}\text { costs(2), emergency department } \\
\text { visits, emergent hospital } \\
\text { admissions(2), length of stay, } \\
\text { diabetes recognition, mental } \\
\text { health(2), pain, perception of } \\
\text { health, well-being, BMI, } \\
\text { triglyceride concentrations, } \\
\text { recovery, clinical outcomes, } \\
\text { self-reported health }\end{array}$ & $\begin{array}{l}\text { smoking, BMI, hypertension, } \\
\text { hypercholesterolaemia, } \\
\text { self-reported health, glycaemic } \\
\text { control, diabetes control, } \\
\text { frequency of hypoglycaemic } \\
\text { reactions, blood sugar, weight }\end{array}$ & \\
\hline $\begin{array}{l}\text { Arbuthnott } \\
\text { et }\left.a\right|^{30}\end{array}$ & $\begin{array}{l}\text { Meta analysis, } \\
\text { 1955-2007, } \\
\text { All } 48 \text { studies } \\
\text { included }\end{array}$ & $\begin{array}{l}\text { Range of } \\
\text { settings }\end{array}$ & $\begin{array}{l}\text { Asthma, bacterial } \\
\text { infection, flbromyalgia, } \\
\text { diabetes, renal } \\
\text { disease, } \\
\text { hypertension, } \\
\text { congestive heart } \\
\text { failure, inflammatory } \\
\text { bowel disease, breast } \\
\text { cancer, HIV and } \\
\text { tuberculosis }\end{array}$ & $\mathrm{P}$ & $\begin{array}{l}\text { Physician-patient } \\
\text { collaboration } \\
\text { (Observation, } \\
\text { surveys) }\end{array}$ & $\begin{array}{l}\text { Medication adherence, } \\
\text { behavioural adherence }\end{array}$ & Appointment adherence & $2 / 1$ \\
\hline Stewart $^{75}$ & $\begin{array}{l}1983-1993 \\
21 \text { studies }\end{array}$ & $\begin{array}{l}\text { Range of } \\
\text { settings }\end{array}$ & $\begin{array}{l}\text { Peptic ulcers, breast } \\
\text { cancer, diabetes, } \\
\text { hypertension, } \\
\text { headache, coronary } \\
\text { artery disease, } \\
\text { gingivitis, tuberculosis, } \\
\text { prostate cancer }\end{array}$ & $\mathrm{P}$ & $\begin{array}{l}\text { Physician-patient } \\
\text { communication } \\
\text { (surveys, evaluation } \\
\text { of audio- or } \\
\text { videotape recording) }\end{array}$ & $\begin{array}{l}\text { Peptic ulcer physical limitation, } \\
\text { blood glucose levels, blood } \\
\text { pressure, headache resolution, } \\
\text { physician evaluation of } \\
\text { symptom resolution for coronary } \\
\text { artery disease, gingivitis and } \\
\text { tuberculosis, anxiety level in } \\
\text { gynaecological care, radiation } \\
\text { therapy, breast cancer care, } \\
\text { functional status following } \\
\text { radiation therapy for prostate } \\
\text { cancer, anxiety after radiation } \\
\text { therapy, pain levels and hospital } \\
\text { length of stay after } \\
\text { intra-abdominal surgery, } \\
\text { physical and psychological } \\
\text { complaints in breast cancer care }\end{array}$ & Details not included & $16 / 5$ \\
\hline $\begin{array}{l}\text { Zolnierek and } \\
\text { DiMatteo }^{28}\end{array}$ & & $\begin{array}{l}\text { Range of } \\
\text { settings }\end{array}$ & $\begin{array}{l}\text { No specific disease } \\
\text { focus }\end{array}$ & $\mathrm{P}$ & $\begin{array}{l}\text { Physician-patient } \\
\text { communication }\end{array}$ & $\begin{array}{l}\text { Adherence to treatment } \\
\text { recommended by clinician }\end{array}$ & $\begin{array}{l}\text { Adherence (2 observational } \\
\text { studies) }\end{array}$ & $125 / 2$ \\
\hline
\end{tabular}


Table 6 Continued

\begin{tabular}{|c|c|c|c|c|c|c|c|c|}
\hline Authors & $\begin{array}{l}\text { Time span and } \\
\text { studies } \\
\text { meeting } \\
\text { inclusion } \\
\text { criteria }\end{array}$ & $\begin{array}{l}\text { Healthcare } \\
\text { setting }\end{array}$ & $\begin{array}{l}\text { Disease areas } \\
\text { covered }\end{array}$ & $\begin{array}{l}\text { Unit of } \\
\text { analysis }\end{array}$ & $\begin{array}{l}\text { Patient experience } \\
\text { focus (and } \\
\text { measurement } \\
\text { methods) }\end{array}$ & $\begin{array}{l}\text { Safety and effectiveness } \\
\text { measure-association } \\
\text { demonstrated - }\end{array}$ & $\begin{array}{l}\text { Safety and effectiveness } \\
\text { measure-association not } \\
\text { demonstrated }\end{array}$ & $\begin{array}{l}\text { Assocs } \\
\text { found vs } \\
\text { not } \\
\text { found } \\
\end{array}$ \\
\hline & $\begin{array}{l}\text { Meta analysis } \\
1949-2008 \\
127 \text { studies }\end{array}$ & & & & $\begin{array}{l}\text { (observation, } \\
\text { surveys) }\end{array}$ & & & \\
\hline Beck et $a l^{76}$ & $\begin{array}{l}1975-2000 \\
5 \text { of } 14\end{array}$ & $\begin{array}{l}\text { Primary } \\
\text { care }\end{array}$ & $\begin{array}{l}\text { No specific disease } \\
\text { focus }\end{array}$ & $P$ & $\begin{array}{l}\text { Physician-patient } \\
\text { communication } \\
\text { (observation, } \\
\text { evaluation of audio } \\
\text { and video tapes) }\end{array}$ & $\begin{array}{l}\text { Compliance with doctors' } \\
\text { advice, blood pressure, pill } \\
\text { count }\end{array}$ & None & $10 / 0$ \\
\hline $\begin{array}{l}\text { Cabana and } \\
\text { Lee }^{21}\end{array}$ & $\begin{array}{l}1966-2002 \\
7 \text { of } 18\end{array}$ & $\begin{array}{l}\text { Range of } \\
\text { settings }\end{array}$ & $\begin{array}{l}\text { Rheumatoid arthritis, } \\
\text { epilepsy, breast } \\
\text { cancer, cervical } \\
\text { cancer, diabetes }\end{array}$ & $P$ & $\begin{array}{l}\text { Continuity of care } \\
\text { (validated measures } \\
\text { of continuity eg, } \\
\text { SCOC) }\end{array}$ & $\begin{array}{l}\text { Hospitalisations, length of stay, } \\
\text { emergency department visits, } \\
\text { intensive care days, preventive } \\
\text { medicine visits, drug or alcohol } \\
\text { abuse, outpatient attendance, } \\
\text { glucose control for adults with } \\
\text { diabetes }\end{array}$ & None & $18 / 5$ \\
\hline $\begin{array}{l}\text { Richards } \\
\text { et } a l^{77}\end{array}$ & $\begin{array}{l}1997-2002, \\
2 \text { of } 33\end{array}$ & $\begin{array}{l}\text { Range of } \\
\text { settings }\end{array}$ & Psoriasis & $P$ & $\begin{array}{l}\text { Patient's perception } \\
\text { of care, satisfaction, } \\
\text { interpersonal skills } \\
\text { (surveys, interviews) }\end{array}$ & $\begin{array}{l}\text { Treatment adherence, } \\
\text { medication use }\end{array}$ & None & $2 / 0$ \\
\hline
\end{tabular}

BMI, body mass index. 
and safety and that focusing on improving patient experience will increase the likelihood of improvements in the other two domains. ${ }^{3}$

The evidence collated in this study demonstrates positive associations between patient experience and the other two domains of quality. Because associations do not entail causality, this does not necessarily prove that improvements in patient experience will cause improvements in the other two domains. However, the weight of evidence across different areas of healthcare indicates that patient experience is clinically important. There is also some evidence to suggest that patients can be used as partners in identifying poor and unsafe practice and help enhance effectiveness and safety. This supports the argument that the three dimensions of quality should be looked at as a group and not in isolation. Clinicians should resist sidelining patient experience measures as too subjective or mood-orientated, divorced from the 'real' clinical work of measuring and delivering patient safety and clinical effectiveness.

Acknowledgements The authors of this work thank Mandy Wearne at NHS Northwest who commissioned this work and provided comments on earlier drafts, We are also grateful to Jocelyn Cornwell who provided comments on an early draft of this article. This article presents independent research commissioned by the National Institute for Health Research (NIHR) under the Collaborations for Leadership in Applied Health Research and Care (CLAHRC) programme for North West London. The views expressed in this publication are those of the author(s) and not necessarily those of the NHS, the NIHR or the Department of Health.

Contributors CD and DB conceived of the study and were responsible for the design and search strategy. CD and LL were responsible for conducting the search. CD and LL conducted the data analysis and produced the tables and graphs. Derek Bell provided input into the data analysis and interpretation. The initial draft of the manuscript was prepared by $C D$ then circulated among all authors for critical revision. All authors helped to evolve analysis plans, interpret data and critically revise successive drafts of the manuscript.

Funding This research received no specific grant from any funding agency in the public, commercial or not-for-profit sectors.

Competing interests None.

Provenance and peer review Not commissioned; externally peer reviewed.

Data sharing statement There are no additional data available.

\section{REFERENCES}

1. Institute of Medicine. Crossing the quality chasm: a new health system for the 21st century. Washington, DC: National Academy Press, 2001.

2. Black N, Jenkinson $C$. Measuring patients experiences and outcomes. BMJ 2009;339:202-5.

3. Department of Health. Liberating the NHS:transparency in outcomes-a framework for the NHS: Department of Health, 2010.

4. Darzi A. High quality care for all-NHS Next Stage Review Final Report. Department of Health, 2008.

5. Department of Health. Using the Commissioning for Quality and Innovation (CQUIN) payment framework, 2008.

6. Berwick DM. What 'patient-centered' should mean: confessions of an extremist. Health Aff 2009;28:w555-65.

7. Street RL, Makoul G, Arora NK, et al. How does communication heal? Pathways linking clinician-patient communication to health outcomes. Patient Ed Couns 2009;74:295-301.

8. Thom DH, Hall MA, Pawlson LG. Measuring patients' trust in physicians when assessing quality of care. Health Aff 2004;23:124-32.

9. Vincent CA, Coulter A. Patient safety: what about the patient? Qual Saf Health Care 2002;11:76-80.
10. Coulter A. Engaging patients in healthcare. Maidenhead, Berkshire: Open University Press, 2011.

11. Rathert C, Huddleston N, Pak Y. Acute care patients discuss the patient role in patient safety. Health Care Manag Rev 2011;36:134-44. 10.1097/HMR.0b013e318208cd31.

12. Picker Institute. Patient experience surveys: the rationale Picker Institute Europe, Oxford, 2008.

13. NICE. Patient experience in adult NHS services: improving the experience of care for people using adult NHS services. Manchester: NICE, 2011.

14. Iles V, Vaughan Smith J. Working in health care could be one of the most satisfying jobs in the world-why doesn't it feel like that?, 2009.

15. López L, Weissman JS, Schneider EC, et al. Disclosure of hospital adverse events and its association with patients' ratings of the quality of care. Arch Intern Med 2009;169:1888-94.

16. Safran DG, Taira DA, Rogers $\mathrm{WH}$, et al. Linking primary care performance to outcomes of care. J Fam Pract 1998;47:213-20.

17. Robert Wood Johnson Foundation. Good for Health, good for business: the case for measuring patient experience of care: The Center for Health Care Quality at the George Washington University Medical Center, Washington DC.

18. Greenhalgh T, Peacock R. Effectiveness and efficiency of search methods in systematic reviews of complex evidence: audit of primary sources. BMJ 2005;331:1064-65.

19. Fuertes J, Boylan L, Fontanella J. Behavioral indices in medical care outcome: the working alliance, Adherence, and related factors. $J$ Gen Intern Med 2009;24:80-5.

20. Raiz LR, Kilty KM, Henry ML, et al. Medication compliance following renal transplantation. Transplantation 1999;68:51-5.

21. Cabana $\mathrm{M}$, Jee $\mathrm{S}$. Does continuity of care improve patient outcomes? J Fam Pract 2004;53.

22. Plomondon M, Magid D, Masoudi F, et al. Association between angina and treatment satisfaction after myocardial infarction. J Gen Intern Med 2008;23:1-6.

23. Jha AK, Orav EJ, Zheng J, et al. Patients' perception of hospital care in the United States. N Engl J Med 2008;359:1921-31.

24. Sequist, et al. Quality Monitoring of Physicians: Linking Patients' Experiences of Care to Clinical Quality and Outcomes. J Gen Intern Med 2008;23.

25. Kaplan SH, Greenfield S, Ware JE. Assessing the effects of physician-patient interactions on the outcomes of chronic disease. Med Care 1989;27(3 Suppl):S110-27.

26. Meterko M, Wright S, Lin $\mathrm{H}$, et al. Mortality among patients with acute myocardial infarction: the influences of patient-centered care and evidence-based medicine. Health Serv Res 2010;45 (5p1):1188-204.

27. Glickman SW, Boulding W, Manary $M$, et al. Patient satisfaction and its relationship with clinical quality and inpatient mortality in acute myocardial infarction. Circ Cardiovasc Qual Outcomes 2010;3:188-95.

28. Zolnierek HKB, DiMatteo MR. Physician communication and patient adherence to treatment: a meta-analysis. Med Care 2009;47:826-34.

29. Drotar D. Physician behavior in the care of pediatric chronic illness: association with health outcomes and treatment adherence. J Dev Behav Pediatr 2009;30:246-54.

30. Arbuthnott A, Sharpe D. The effect of physician-patient collaboration on patient adherence in non-psychiatric medicine. Patient Ed Couns 2009;77:60-7.

31. Lewis ET, Combs A, Trafton JA. Reasons for under-use of prescribed opioid medications by patients in pain. Pain Med 2010;11:861-71.

32. Kahn KL, Schneider EC, Malin JL, et al. Patient centered experiences in breast cancer: predicting long-term adherence to tamoxifen use. Med Care 2007;45:431-9.

33. Schneider EC, Zaslavsky AM, Landon BE, et al. National quality monitoring of medicare health plans: the relationship between enrollees' reports and the quality of clinical care. Med Care 2001;39:1313-25.

34. Schoenthaler A, Chaplin WF, Allegrante JP, et al. Provider communication effects medication adherence in hypertensive African Americans. Patient Ed Couns 2009;75:185-91.

35. Heisler M, Bouknight RR, Hayward RA, et al. The relative importance of physician communication, participatory decision making, and patient understanding in diabetes self-management. J Gen Intern Med 2002;17:243-52.

36. Haynes RB, Ackloo E, Sahota N, et al. Interventions for enhancing medication adherence. Cochrane Database Syst Rev 2008;2.

37. Flocke SA, Stange KC, Zyzanski SJ. The association of attributes of primary care with the delivery of clinical preventive services. Med Care 1998;36:AS21-30. 
38. O'Malley AS, Sheppard VB, Schwartz M, et al. The role of trust in use of preventive services among low-income African-American women. Prev Med 2004;38:777-85.

39. Carcaise-Edinboro P, Bradley CJ. Influence of patient-provider communication on colorectal cancer screening. Med Care 2008;46:738-45.

40. Jackson CA, Clatworthy J, Robinson A, et al. Factors associated with non-adherence to oral medication for inflammatory bowel disease: a systematic review. Am J Gastroenterol 2010;105:525-39.

41. Clark NM, Cabana MD, Nan B, et al. The clinician-patient partnership paradigm: outcomes associated with physician communication behavior. Clin Pediatr 2008;47:49-57.

42. Stewart M, Brown J, Donner A, et al. The impact of patient-centered care on outcomes. J Fam Pract 2000;49:796-804.

43. Sans-Corrales M, Pujol-Ribera E, GenÃ@-Badia J, et al. Family medicine attributes related to satisfaction, health and costs. Fam Pract 2006;23:308-16.

44. Saultz JW, Lochner J. Interpersonal continuity of care and care outcomes: a critical review. Ann Fam Med 2005;3:159-66.

45. Hsiao C-J, Boult C. Effects of quality on outcomes in primary care: a review of the literature. A J Med Qual 2008;23:302-10.

46. Isaac T, Zaslavsky AM, Cleary PD, et al. The relationship between patients' perception of care and measures of hospital quality and safety. Health Serv Res 2010;45:1024-40.

47. Rao M, Clarke A, Sanderson C, et al. Patients' own assessments of quality of primary care compared with objective records based measures of technical quality of care: cross sectional study. BMJ 2006;333:19-22.

48. Chang JT, Hays RD, Shekelle PG, et al. Patients' global ratings of their health care are not associated with the technical quality of their care. Ann Intern Med 2006;145:635-6.

49. Weingart SN, Pagovich O, Sands DZ, et al. What can hospitalized patients tell us about adverse events? Learning from patient-reported incidents. J Gen Intern Med 2005;20:830-6.

50. Weissman JS, Schneider EC, Weingart SN, et al. Comparing patient-reported hospital adverse events with medical record review: do patients know something that hospitals do not? Ann Intern Med 2008;149:100-8.

51. Solberg LI, Asche SE, Averbeck BM, et al. Can patient safety be measured by surveys of patient experiences? Jt Comm J QualPatient Saf 2008;34:266-74.

52. O'Connor AM, Bennett CL, Stacey D, et al. Decision aids for people facing health treatment or screening decisions. Cochrane database Syst Rev 2009(3):CD001431.

53. Mumford E, Schlesinger HJ, Glass GV. The effect of psychological intervention on recovery from surgery and heart attacks: an analysis of the literature. Am J Public Health 1982;72:141-51.

54. Begg C, Berlin J.N.J. Publication bias: a problem in interpreting medical data. J R Stat SocSer A 1988;151.

55. Burgers JS, Voerman GE, Grol R, et al. Quality and coordination of care for patients with multiple conditions: results from an international survey of patient experience. Eval Health Prof 2010;33:343-64.

56. Vincent $\mathrm{C}$. Understanding and responding to adverse events. $\mathrm{N}$ Engl J Med 2003;348:1051-6.

57. Agoritsas T, Bovier PA, Perneger TV. Patient reports of undesirable events during hospitalization. J Gen Intern Med 2005;20:922-8.

58. Jackson JL, Chamberlin J, Kroenke K. Predictors of patient satisfaction. Soc Sci Med 2001;52:609-20.
59. Safran DG, Miller W, Beckman H. Organizational dimensions of relationhip-centred care. J Gen Intern Med 2005;21:S9-15.

60. Alamo MMo, Moral RR, PÃ@rula de Torres LA. Evaluation of a patient-centred approach in generalized musculoskeletal chronic pain/fibromyalgia patients in primary care. Patient Educ Couns 2002;48:23-31.

61. Fan VS, Reiber GE, Diehr P, et al. Functional status and patient satisfaction. J Gen Intern Med 2005;20:452-9.

62. Little $\mathrm{P}$, Everitt $\mathrm{H}$, Williamson I, et al. Observational study of effect of patient centredness and positive approach on outcomes of general practice consultations. BMJ 2001;323:908-11.

63. Levinson W, Roter DL, Mullooly JP, et al. Physician-patient communication: the relationship with malpractice claims among primary care physicians and surgeons. JAMA1997;277:553-9.

64. Slatore, Christopher G, Cecere, et al. Patient-clinician communication: associations with important health outcomes among veterans with COPD. Northbrook, ETATS-UNIS: American College of Chest Physicians, 2010.

65. Lee $Y-Y$, Lin JL. The effects of trust in physician on self-efficacy, adherence and diabetes outcomes. Soc Sci Med 2009;68: 1060-8.

66. Lee $Y-Y$, Lin JL. Do patient autonomy preferences matter? Linking patient-centered care to patient-physician relationships and health outcomes. Soc Sci Med 2010;71:1811-18.

67. Kennedy A, Nelson E, Reeves D, et al. A randomised controlled trial to assess the impact of a package comprising a patient-orientated, evidence-based self-help guidebook and patient-centred consultations on disease management and satisfaction in inflammatory bowel disease. Health Technol Assess (Winchester, England) 2003;7:iii, 1-113.

68. Kinnersley P, Stott N, Peters TJ, et al. The patient-centredness of consultations and outcome in primary care. Br J Gen Pract 1999:49:711-16.

69. Fremont A, Cleary P, Hargraves J, et al. Patient-centered processes of care and long-term outcomes of myocardial infarction. $J$ Gen Intern Med 2001:16:800-8.

70. Riley DL, Stewart DE, Grace SL. Continuity of cardiac care: cardiac rehabilitation participation and other correlates. Int J Cardiol 2007:119:326-33.

71. Blasi ZD, Harkness E, Ernst E, et al. Influence of context effects on health outcomes: a systematic review. Lancet 2001;357:757-62.

72. Hall AM, Ferreira PH, Maher CG, et al. The influence of the therapist-patient relationship on treatment outcome in physical rehabilitation: a systematic review. Phys Ther 2010;90:1099-110.

73. Stevenson FA, Cox K, Britten N, et al. A systematic review of the research on communication between patients and health care professionals about medicines: the consequences for concordance. Health Expect 2004;7:235-45.

74. Hall JA, Roter DL, Katz NR. Meta-analysis of correlates of provider behavior in medical encounters. Med Care 1988;26:657-75.

75. Stewart MA. Effective physician-patient communication and health outcomes: a review. Can Med Assoc J 1995;152:1423-33.

76. Beck RS, Daughtridge R, Sloane PD. Physician-patient communication in the primary care office: a systematic review. $J A m$ Board Famy Pract 2002;15:25-38.

77. Richards HL, Fortune DG, Griffiths CEM. Adherence to treatment in patients with psoriasis. J Eur Acad Dermatol Venereol 2006;20:370-9. 